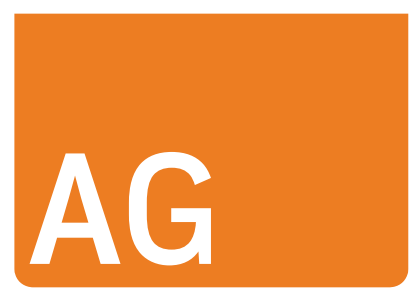

\title{
Die Aktiengesellschaft
}

Zeitschrift für deutsches, europäisches und internationales Aktien-, Unternehmensund Kapitalmarktrecht

\section{Herausgeber:}

Prof. Dr. Heinz-Dieter Assmann, LL.M., Universität Tübingen, E-Mail: assmann@jura.uni-tuebingen.de · Prof. Dr. Mathias Habersack, Universität München, E-Mail: mathias.habersack@jura.uni-muenchen.de

in Verbindung mit VorsRiBGH Prof. Dr. Ingo Drescher, Karlsruhe · Prof. Dr. Volker Emmerich, Bayreuth . VizepräsBVerfG Prof. Dr. Stephan Harbarth, LL.M., Karlsruhe · Prof. Dr. Jens Koch, Bonn · Prof. Dr. Hans-Joachim Mertens, Königstein · Prof. Dr. Dr. h.c. Uwe H. Schneider, Mainz/Frankfurt a.M. · RA Prof. Dr. Jochen Vetter, München · Prof. Dr. Dr. h.c. Wolfgang Zöllner, Tübingen

\section{Ständige Mitarbeiter AG-Report:}

RA Prof. Dr. Michael Arnold, Stuttgart · Prof. Dr. Walter Bayer, Jena · Dipl.-Verw. Wiss. Marianne Gajo, Spaichingen · Dr. Thomas Ledermann, Hamburg · Dr. Franz-Josef Leven, Frankfurt a.M. · Dr. Stefan Mai, Frankfurt a.M. · RA Dr. Stefan Mutter, Düsseldorf · Markus Rieger, Wolfratshausen · WP Prof. Dr. Eberhard Scheffler, Hamburg · Dipl.-Vw. Christoph Schlienkamp, Düsseldorf · Andreas Schmidt, München · Prof. Dr. Dr. h.c. Uwe H. Schneider, Mainz/Frankfurt a.M. · RAin Daniela Weber-Rey, LL.M., Frankfurt a.M. RA Dr. Jochen Weck, München

\section{Steuer-Journal:}

Streck Mack Schwedhelm, Rechtsanwälte/Fachanwälte für Steuerrecht, Köln/Berlin/München

\section{Inhalt}

\section{Aufsätze}

\section{Prof. Dr. Jens Koch - Die Ad-hoc-Publizität: Veröffentlichungs- oder Wissensorganisationspflicht?}

Über Jahrzehnte hinweg führte die Ad-hoc-Publizität ein unbeachtetes Schattendasein. Die Vorgänge am Neuen Markt haben ihr größere Aufmerksamkeit gebracht, aber erst eine vor vier Jahren angestoßene Diskussion um ihr Verständnis als Wissensorganisationspflicht hat sie zu einem der zentralen Regelungskomplexe des Kapitalmarktrechts avancieren lassen. Zugleich hat sich im jüngeren Fallmaterial auch unmittelbar gezeigt, welche verborgene Sprengkraft eine so verstandene Pflicht zur Ad-hoc-Publizität entfalten kann. Vor diesem Hintergrund soll in diesem Beitrag untersucht werden, ob Art. 17 Abs. 1 MMVO tatsächlich eine solche "Compliance-Dimension" beinhaltet oder ob sich sein Regelungsgehalt nicht doch in dem erschöpft, was sein Wortlaut nahelegt: in einer Veröffentlichungspflicht.

RA (SRA) Dr. Richard Backhaus, LL.M. (Edin.)/RA (SRA) Dr. Tobias Brouwer - Zustimmungsvorbehalte des Aufsichtsrats bei Geschäften mit nahestehenden Personen (Related Party Transactions) bei der KGaA - HGB sticht AktG

Anders als der Referentenentwurf zum ARUG II enthält die Begründung zum Regierungsentwurf eine Aussage darüber, inwieweit die Regelungen über das Zustimmungsverfahren bei Related Party Transactions (RPT) auch in der börsennotierten KGaA anzuwenden sind. Danach ist unter Rückgriff auf $§ 278$ Abs. 3 AktG auch in der KGaA der Aufsichtsrat der KGaA für die Zustimmungsentscheidung bei wesentlichen Geschäften mit nahestehenden Personen nach § 111b AktG-RegE zuständig. Nach Auffassung der Verfasser ist das in § 111b AktG-RegE festgelegte Kompetenzverhältnis zwischen Vorstand und Aufsichtsrat jedoch nicht unter $\$ 278$ Abs. 3 AktG, sondern unter das in $\$ 278$ Abs. 2 AktG geregelte Rechtsverhältnis zu subsumieren. In der Folge finden auf die Zustimmungsentscheidung bei Geschäften mit nahestehenden Personen in der KGaA die Vorschriften des HGB über die Kommanditgesellschaft Anwendung, die gemäß § 164 Satz 1 HGB eine Übertragung der Zustimmungskompetenz auf ein fakultatives Gesellschaftsorgan zulassen. 
Inhalt

\section{Kommentar}

RA Dr. Daniel Schubmann / RA Stefan Hubertus Tolksdorf - Die Vertretung der AG bei Unternehmenstransaktionen mit (zukünftigen) Vorstandsmitgliedern - Kommentar zU BGH v. 15.1.2019 - II ZR 392/17, AG 2019, 298

Auf den ersten Blick mag der Sachverhalt, der der Entscheidung des BGH v. 15.1.2019 - II ZR 392/17, AG 2019, 298 - in dieser Ausgabe, zugrunde liegt, etwas außergewöhnlich erscheinen, bei näherer Betrachtung stellt sich diese Konstellation gerade vor dem Hintergrund des "Start-up" Booms als sehr naheliegend dar. Vielfach werden Gründer als wichtig(st)er Unternehmenswert in Konzernstrukturen übernommen, indem sie ihre zumeist junge Gesellschaft übertragen und im Gegenzug in die Vorstandsebene des Käufers eingebunden werden. Die Entscheidung ist aber im Grunde übertragbar auf jedwede Vertragsbeziehung zwischen der AG und einem (zukünftigen) Vorstandsmitglied oder - und das ist bahnbrechend an der vorliegenden Entscheidung - auch an einer Gesellschaft, an der ein Vorstandsmitglied beteiligt ist.

\section{Rechtsprechung}

Aktienrecht: Vertretung der AG bei Rechtsgeschäften mit einer GmbH, deren alleiniger Gesellschafter ein Vorstandsmitglied ist

(BGH, Urt. v. 15.1.2019 - II ZR 392/17).

Umwandlungsrecht: Keine Differenzhaftung der Gesellschafter, aber mögliche Haftung für existenzvernichtenden Eingriff

(BGH, Teilversäumnis- und Teilendurt. v. 6.11.2018 - II ZR 199/17)

Unternehmensbewertung: Bewertung von Versicherungsunternehmen, Anwendung von Multiplikatorverfahren

(OLG Düsseldorf, Beschl. v. 6.9.2018 - 26 W 1/18 (AktE) -

Victoria/Ergo III).

(OLG Köln, Beschl. v. 13.7.2018 - 28 Wx 2/18).

nehmens

(OLG Stuttgart, Beschl. v. 20.8.2018 - 20 W 21/18) 315 sammensetzung des Aufsichtsrats

Societas Europaea: Zusammensetzung des Verwaltungsrats einer Europäischen Aktiengesellschaft, Verbot der Schlechterstellung

(LG Frankfurt/M., Urt. v. 3.5.2018 - 3-05 0 101/17, nrkr.).... 317

\section{Buchbesprechungen}

RA Matthias Höreth - Langenbach, Der Versammlungsleiter in der Aktiengesellschaft - Zurückweisungskompetenz - Abwahl - Haftung ..... 319

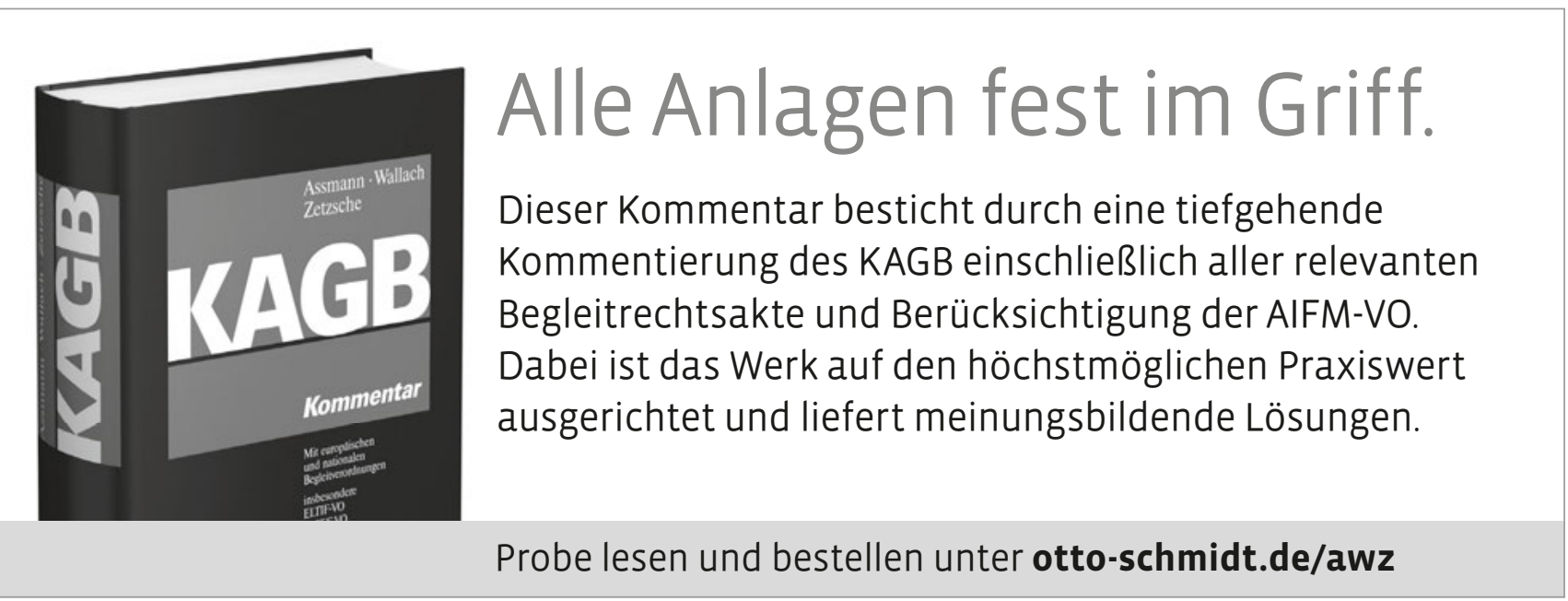


Inhalt

\section{AG Report}

Rechts-Report | Aus der Gesetzgebung

Leitlinien der EU Kommission zur Vergütungsberichterstattung nach ARUG II vor der Türe (Stefan Mutter)......

Rechts-Report | Aktienrecht in Zahlen

Beschlussmängelklagen-Monitor 2017/2018 (Walter Bayer /Thomas Hoffmann).....

Rechts-Report | HV-Praxis

Wird Nachhaltigkeit zum festen Bestandteil auf Hauptversammlungen? (Sandra Reich).....

Kapitalmarkt-Report | Zahlen, Fakten, Entwicklungen

Es bleibt eine komplizierte Beziehung - Die Marktmissbrauchsverordnung sorgt auch nach fast drei Jahren noch für Verunsicherung (Bastian Heidecker).......

Kapitalmarkt-Report | Börse

Britische Aufsicht will Wettbewerb bei Investitionsplattformen verbessern (Marianne Gajo).......................................... Rit20

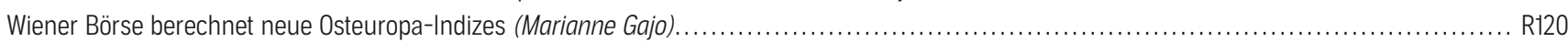

MarketAxess erhält Genehmigung für Handelsplattform in den Niederlanden (Marianne Gajo) .............................................. Rit20

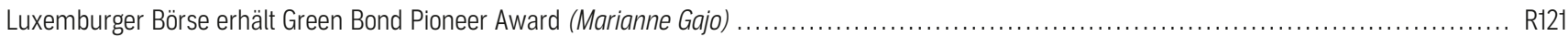

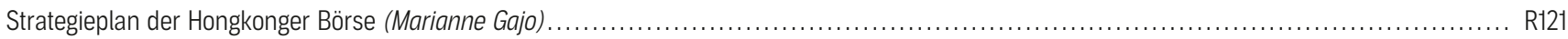

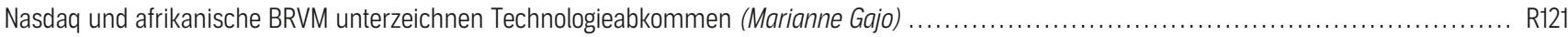

Branchen- und Unternehmens-Report | Branchen-Nachrichten

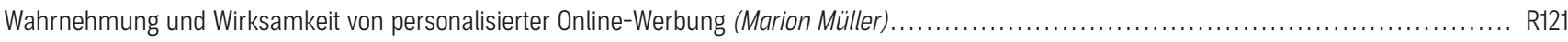

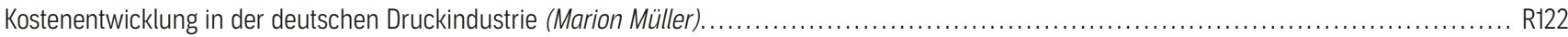

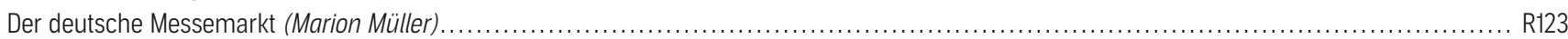

Branchen- und Unternehmens-Report | Jahresabschlüsse

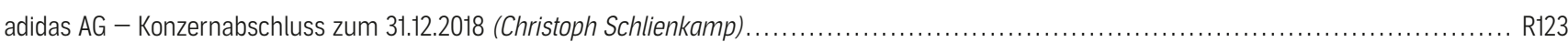

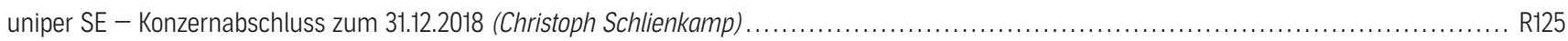

\section{Bibliothek}

Neuerscheinungen (Katharina Melkko)

\section{Wussten Sie schon ...}

Im Beratermodul AG haben Sie Zugriff auf das Online-Archiv Ihrer Zeitschrift. In der Zeitschriften-App lesen Sie aktuelle Beiträge auf Ihrem Smartphone. Bei Fragen zu Ihren Freischaltcodes wenden Sie sich gerne an den Kundenservice: Telefon 0221 / 93738-997 oder E-Mail an kundenservice@otto-schmidt.de.

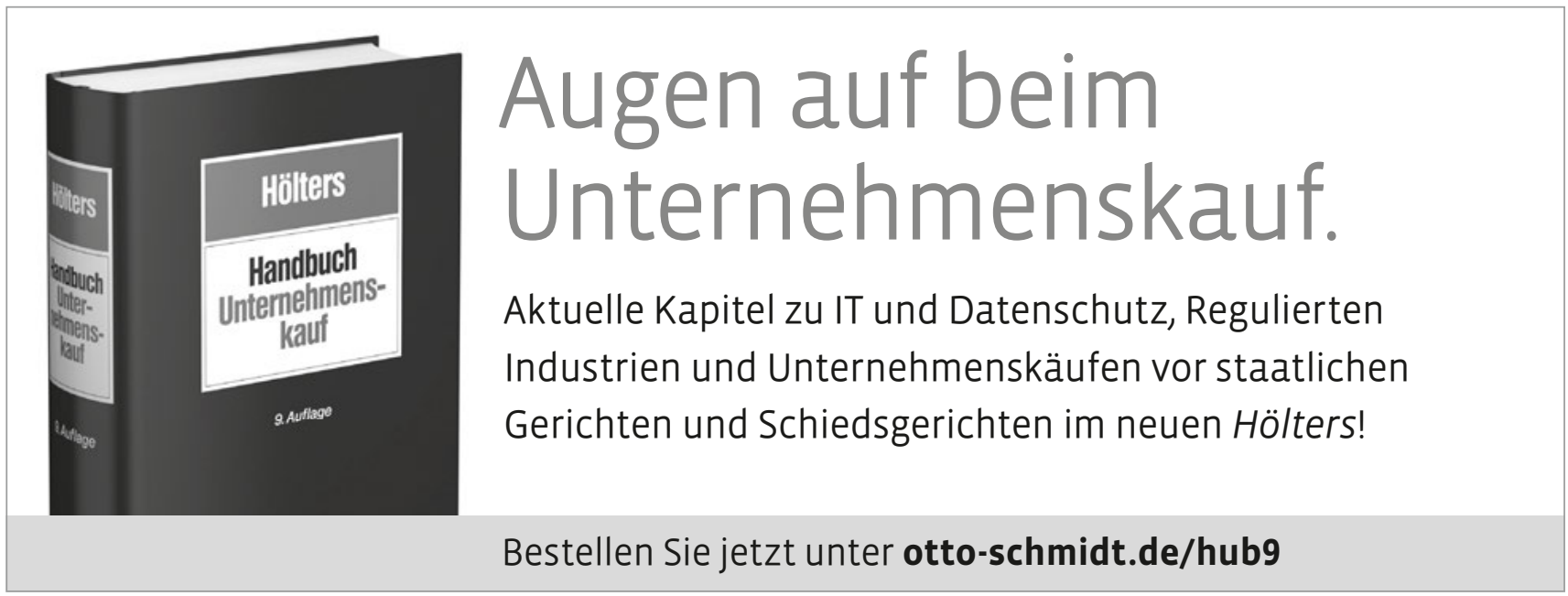

